\title{
MATHEMATICAL MODELING OF SALT LENS FORMATION PROCESS IN THE CATHODE ASSEMBLY OF ALUMINUM ELECTROLYSIS CELLS
}

\author{
Anton Orlov ${ }^{1, *}$, and Alexander Proshkin ${ }^{1}$ \\ ${ }^{1}$ Siberian Federal University, Kirensky st. 26, Krasnoyarsk, Russia
}

\begin{abstract}
In this paper, with the help of mathematical modeling we investigate growth under saline lens bottom blocks of the cathode aluminum cell. The penetration of the melt in the hearth of the cathode of the device is one of the main causes of premature shutdown of cells, because it leads to excessive heat loss and destruction of the hearth. The study was built mathematical model of unsteady filtration and heat the porous body with an incompressible viscous fluid. With the help of the developed mathematical model we studied the dynamics of growth of the lens. Research has identified the rate of melt penetration into the hearth of the cathode unit in the course of its life. The calculation results were compared with the field data.
\end{abstract}

\section{Introduction}

Lifting bottom blocks in industrial aluminum cell caused by several mechanisms. One of the key mechanisms is the formation of the salt of the lens between the bottom blocks and refractory material. The growth of this lens leads to accelerate the erosion of the cathode blocks and consequently decrease the life of the cell. The mechanism of their formation is not fully understood, but the main reason is probably the reaction between the liquid electrolyte is filtered through pores with carbon and molten aluminum, have got through the cracks [1]. Mechanical disruption of the cathode blocks, leading to stop electrolysis will occur if overpressure exceeds the strength of the porous material. Along with this ascent of the cathode blocks may cause substantial deformation of blooms. The greatest deformation normally occur at the point where the blooms coming out of the bottom blocks and where there is also a high enough temperature to make easily deformable steel [2, 3].

Figure 1 clearly shows how the hosted lining materials impregnated with electrolyte. Impregnating the cathode electrolyte block rushes to the refractory materials where there are physical and chemical transformations of these materials. In particular, albite is formed which

\footnotetext{
*Corresponding author: an_riv@mail.ru
} 
is highly viscous glassy substance during these reactions. Formation albite is the primary mechanism that prevents further impregnation materials. When albite forming reaction front reaches the solidus isotherm, the melt cannot penetrate into the bath hearth. He begins to accumulate between the cathode blocks and refractory material, forming a so-called lens. Growth leads to lens ejection cathode blocks leading to their destruction.

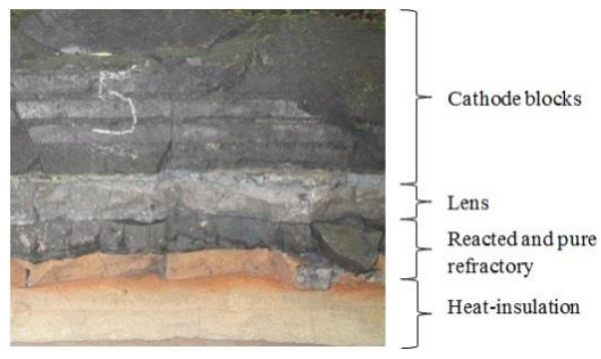

Fig. 1. Spent a cathode assembly (Part).

The aim of this work is the calculation of the growth rate of lens and identifying ways to reduce it by means of mathematical modeling. Mathematical modeling is a valuable tool that can help to optimize the design of the cathode liner device that will minimize lens growth during the entire period of operation of the cell, thus increasing its service life.

During continuous operation of the cell occurs in the electrolyte filtering hearth cathode. The hydrostatic pressure and capillary forces electrolyte displaces air in the pores of lining materials. The melt is introduced through the carbon block and responds within a certain range with a refractory material to form a dense vitreous and impervious barrier at $800-840{ }^{\circ} \mathrm{C}$, which stops or significantly hinders the further introduction of the hearth. In the calculation of this process is taken into account an increase in the viscosity of the electrolyte as it passes through the refractory layer.

\section{Statement of the problem}

This task is considered a two-phase filtration of incompressible immiscible liquids in a heterogeneous non-deformable porous body. It is believed that the dynamic viscosity of the phases are constant, fluid flow is slow and there is no phase transition.

Table 1. Initial data.

\begin{tabular}{|l|c|c|}
\hline Temperature of the electrolyte & ${ }^{\circ} \mathrm{C}$ & 956 \\
\hline Temperature of the environment & ${ }^{\circ} \mathrm{C}$ & 20 \\
\hline Temperature of the electrolyte solidus & ${ }^{\circ} \mathrm{C}$ & 820 \\
\hline Heat transfer coefficient: & & 830 \\
melt / unit & $\mathrm{W} /\left(\mathrm{m}^{2} \cdot{ }^{\circ} \mathrm{C}\right)$ & 25 \\
cover / air & $\mathrm{W} /\left(\mathrm{m}^{2} \cdot{ }^{\circ} \mathrm{C}\right)$ & 2100 \\
\hline The density of the electrolyte & $\mathrm{kg} / \mathrm{m}^{3}$ & $2,79 \cdot 10^{-3}$ \\
\hline The viscosity of the electrolyte & $\mathrm{Pa} \cdot \mathrm{s}$ & $10^{-13}$ \\
\hline The permeability of the bottom blocks & $\mathrm{m}^{2}$ & \\
\hline
\end{tabular}


The computational domain is a half portion of the cathode unit of the cell geometry is shown in figure 2. The initial data are given in Table 1 . The weakest point in the design of the cell are printed interconnects and peripheral joints, so the simulation was carried out taking into account these features. The calculation takes into account all the components of an aluminum electrolytic this design, including interconnect joints. electrolyte filtration occurs through the porous structure of the hearth block and interconnect joints.

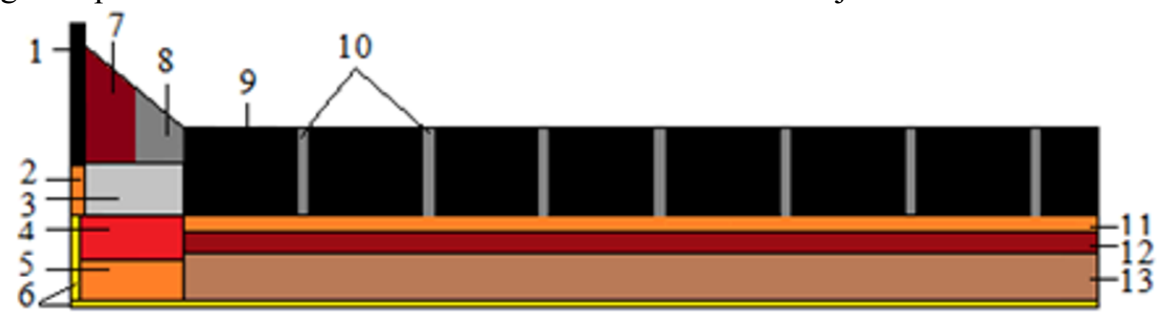

Fig 2. The geometry of the computational domain: 1 - SiC block, 2 - compensator, 3 - refractory concrete, 4 - refractory, 5 - heat insulation, 6 - leveling backfilling, 7 - inserter, 8 - peripheral seam, 9 bottom block, 10 - interblock seams, 11 and 12 - refractory, 13 - heat insulation.

\section{Mathematical model}

Mathematical model of non-stationary filtration process and porous body heat transfer with incompressible viscous liquid consist of following equation system.

- liquid-flow equation in porous medium

$$
\vec{w}=-[D] \operatorname{grad} P
$$

- liquid continuity equation

$$
\frac{\partial \varepsilon \rho}{\partial \tau}+\operatorname{div}(\rho \vec{w})=0
$$

- energy equation for liquid

$$
(\rho c)_{G} \varepsilon \frac{\partial T_{G}}{\partial \tau}+\operatorname{div}\left((\rho c)_{G} \vec{w} T_{G}-\lambda_{G} \operatorname{grad} T_{G}\right)=\alpha_{V}\left(T_{S}-T_{G}\right)
$$

Where the first term of equation on the left describes the increase of enthalpy, second term of equation heat convection, and on the right described heat transfer between liquid and solid body:

- heat-transfer equation in the porous medium

$$
(\rho c)_{S}(1-\varepsilon) \frac{\partial T_{S}}{\partial \tau}+\operatorname{div}\left(-[\lambda]_{S} \operatorname{grad} T_{S}\right)=\alpha_{V}\left(T_{G}-T_{S}\right)+q_{V}
$$

where: 


$$
[\lambda]_{G}=\left[\begin{array}{ccc}
\lambda_{1} & 0 & 0 \\
0 & \lambda_{2} & 0 \\
0 & 0 & \lambda_{3}
\end{array}\right]
$$

- effective heat-conduction tensor, recorder in the coordinate system $(\mathrm{X}, \mathrm{Y}, \mathrm{Z}) \mathrm{c}$ coincides with the main axes of the heat conduction, and $q_{V}$ - volumetric heat generation in porous material;

- equation of condition for liquid

$$
\rho_{G}=\rho\left(p, T_{G}, \tau\right)
$$

In this study, to determine the boundaries of the free electrolyte fluid used in the method of cells (VOF), proposed by Hirt and Niholsom [4]. This method is considered to be one of the most effective methods for solving problems with the free surface of the liquid. Application of this method to solve similar problems can be found in $[5,6]$. The idea of this method is that the liquid and gas are considered as a single two-component medium, and the spatial distribution of the phases within the computational domain is defined by a special marker functions $F(x, y, z, t)$, which value is the volume fraction of liquid phase in the computational cell as follows:

$$
f(x)= \begin{cases}0, & \text { if the cell is empty } \\ 1, & \text { if the cell is filled }\end{cases}
$$

and $0<F(x, y, z, t)<1-$ if the phase boundary passes through cell.

Since the free surface of the electrolyte moves with liquid, then tracking free boundary moving in space accomplished by solving transport equation of liquid phase volume ratio in cell.

$$
\frac{\partial \varepsilon F}{\partial t}+\vec{w} \cdot \nabla \varepsilon F=0
$$

Here $\vec{w}$ - velocity vector of two-phase medium, found by solving the system of equations of hydrodynamics. The density and molecular viscosity two-component medium are considered by the volume fraction of the liquid in the cell mixture according to the rule:

$$
\begin{aligned}
& \rho=\rho_{1} F+(1-F) \rho_{2}, \\
& \mu=\mu_{1} F+(1-F) \mu_{2} .
\end{aligned}
$$

Here $\rho_{1}, \mu_{1}-$ density and viscosity of electrolyte, $\rho_{2}, \mu_{2}-$ respectively, density and viscosity of air. Thus obtained density values $\rho$ and viscosity $\mu$ are included in the equation of motion and determine the physical properties of the two-phase medium. 


\section{Results and discussion}

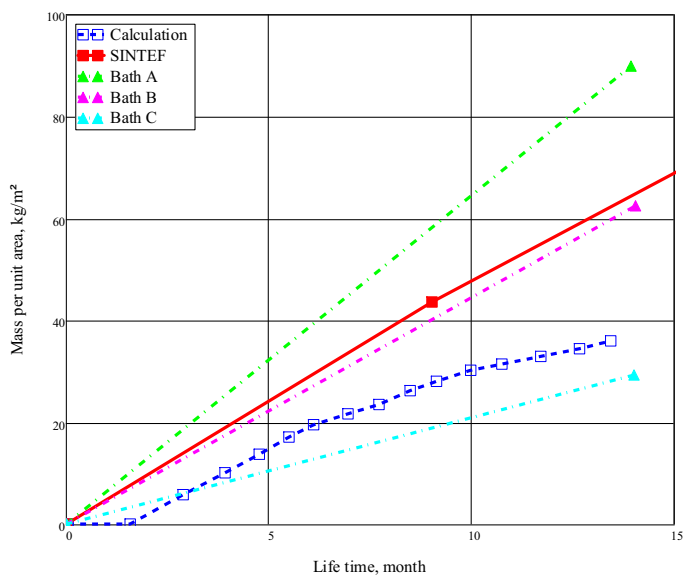

Fig. 3. Dynamics of the melt in the cathode assembly.

Figure 3 shows a comparison of the calculated results to the rate of arrival of the melt into the refractory material of the cathode device with the experimental data obtained at the Norwegian Institute of Technology (SINTEF) and field data on the melt flow of some industrial baths (A, B, C). Since the baths are different in size, comparison of data produced by the melt mass, coming through the unit area. It can be seen that the calculation agrees qualitatively with the field data. Also on the calculated line it is seen that the active electrolyte penetration into the refractory material begins after two months of operation of the cell. During this time, the electrolyte is impregnated only bottom blocks. Modeling in this way makes it possible to predict only 1,5 years of use of baths.

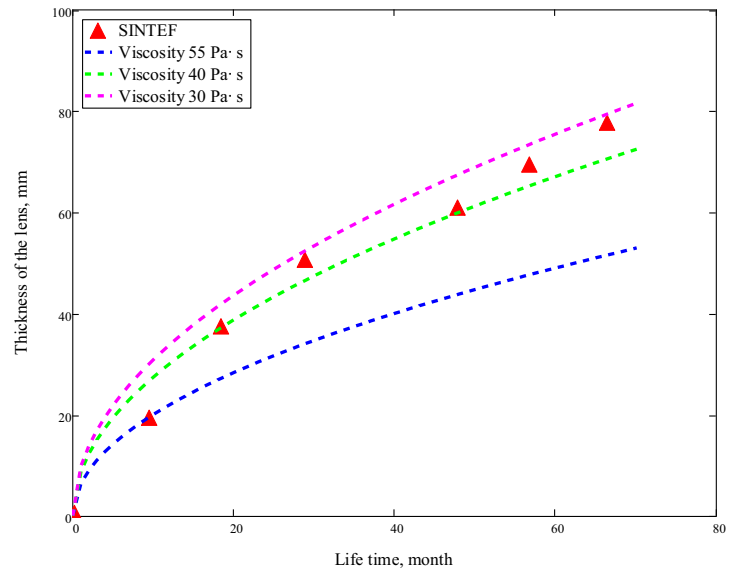

Fig 4. The dynamics of growth of the lens.

Figure 4 shows a comparison of the calculation results with the experimental data for SINTEF lens growth with electrolyte penetration speed in the hearth of the cell. The viscosity 
of the electrolyte was varied between 30 and $55 \mathrm{~Pa} \cdot \mathrm{s}$. It is evident that the calculations carried out in this way allows a fairly high quality characterize the dynamics of the growth of the lens

\section{Conclusions}

A mathematical model of the lens of the growth process. The comparison of the calculation results with the field data. With the help of the developed mathematical models of the dynamics of the impregnation of the lining of the cathode electrolyte device. Studies have shown a dynamic melt infiltration hearth bath during operation of the cell. Further results will evaluate the thermal processes in the electrolyte penetration electrolyzer cathode lining materials, and enable the liner to optimize the design to reduce costs and increase the thermal lifetime of the cathode device.

The developed mathematical model allows to simulate thermal processes and electrolyte penetration education lens up to 1.5 years of operation of the cell. To predict the dynamics of the growth of the lens after one and a half years require adjustment model, which would allow to carry out changes in the computational domain in the simulation.

\section{References}

1. M. Sorlie, H.A. Oye, Cathodes in Aluminium Electrolysis. 2nd edition (AluminiumVertag, Düsseldorf, 1994)

2. S. Pietrzyk, P. Palimaka, W. Gebarowski, Arch. Metall. Mater. 59 (2014)

3. Femi Fakoya, Donald Picard. Characterisation of the material behaviour of cathode steelcollector bar at high temperatures and low stress levels (Light Metals, 2014)

4. C.W. Hirt, B.D. Nichols, J. Comput. Phys. 39 (1981)

5. A.A. Shebeleva, A.V. Minakov, A.A. Yagodnitsina, V.G. Andyuseva. MATEC Web Conf. 23 (2015)

6. A.S. Orlov, A.V. Minakov, A.V. Proshkin. MATEC Web Conf. 23 (2015) 